\title{
Fetal monitoring and anaesthesia for fetal distress
}

\section{Fetal monitoring}

Should all parturients have continuous electronic fetal heart rate (FHR) monitoring during labour? Large prospective, randomized studies suggest that there is little or no benefit to the universal use of continuous, electronic FHR monitoring during labour. ${ }^{1-9}$ Further, some studies suggest that electronic FHR monitoring results in an increased incidence of Caesarean section. ${ }^{1,3,4,7}$ Guidelines for Perinatal Care, third edition, ${ }^{10}$ includes the following statement regarding FHR monitoring for high-risk patients: "It has been shown that with a 1:1 nurse/patient ratio, intermittent auscultation at intervals of 15 minutes during the active phase of the first stage of labor and 5 minutes during the second stage is equivalent to continuous electronic fetal heart rate monitoring."

It is unlikely, however, that many obstetricians in the United States will abandon the use of universal, continuous electronic FHR monitoring. Few labour and delivery units in the United States can consistently satisfy the nursing standards required for rigorous, intermittent auscultation of the FHR. Further, the fear of litigation prompts many obstetricians to use electronic FHR monitoring in their patients.

Most of the aforementioned studies were performed in hospitals with infrequent utilization of epidural anaesthesia during labour. Thus, these studies may not be applicable for women receiving regional anaesthesia during labour. Neither the American Society of Anesthesiologists (ASA) nor the American College of Obstetricians and Gynecologists (ACOG) has issued specific guidelines regarding FHR monitoring in patients receiving regional anaesthesia. The ASA" has stated: "Regional anesthesia for labor and/or vaginal delivery requires that the parturient's vital signs and the fetal heart rate be monitored and documented by a qualified individual. Additional monitoring appropriate to the clinical condition of the parturient and the fetus should be employed when indicated." The $\mathrm{ACOG}^{12}$ has stated: "When maternal hemodynamics are changing, such as with maternal hypotension from conduction anesthesia or maternal hemorrhage, increased fetal surveillance may be indicated." (Of course, one cannot predict which patients will experience hypotension during regional anaesthesia.) I prefer that my patients have continuous electronic FHR monitoring before, during, and after induction of epidural anaesthesia during labour.

\section{Anaesthesia for fetal distress}

The presence of fetal distress represents high medical risk for the fetus and the mother, and high medico-legal risk for the obstetrician and the anaesthetist. Chadwick et al. ${ }^{13}$ reviewed malpractice claims filed against anesthetists for obstetric cases. They compared obstetric versus nonobstetric claims. Maternal or patient death was the leading complication in both obstetric and non-obstetric claims, but in the obstetric claims, "newborn brain damage was almost as common as maternal death." Further, the authors noted: "Although adverse newborn outcome was judged not to be related to anesthetic care in half of the cases, newborn brain injury was a leading cause of claims against obstetric anesthesiologists." ${ }^{13}$

Recently the ACOG Committee on Obstetrics: Maternal and Fetal Medicine issued a committee opinion entitled: "Anesthesia for Emergency Deliveries." 14 This opinion is sympathetic to the concerns of anaesthetists. I should like to summarize that opinion as follows:

1 The ACOG acknowledges that failed intubation and pulmonary aspiration of gastric contents remain prominent causes of maternal morbidity and mortality from anaesthesia.

2 The ACOG recommends that "the obstetric care team should be alert to the presence of risk factors that place the parturient at increased risk for complications from emergency general or regional anesthesia."

3 When risk factors are identified, ACOG recommends that the obstetrician obtain antepartum consultation from an anaesthetist. (For this recommendation to be effective, anaesthetists should establish procedures to facilitate antepartum consultation.)

4 The ACOG recommends that the obstetrician and anaesthetist develop "strategies ... to minimize the need for emergency induction of general anesthesia in women for whom this would be especially hazardous."

From the Department of Anesthesia and Obstetrics and Gynecology, University of Iowa College of Medicine, Iowa City, Iowa 52242. 
Such strategies might include early establishment of intravenous access and early placement of an epidural or spinal catheter.

5 In emergency obstetric cases, ACOG acknowledges that "the maternal as well as the fetal status must be considered." They further state: "Although there are some situations in which general anesthesia is preferable to regional anesthesia, the risk of general anesthesia must be weighed against the benefit for those patients who have a greater potential for complications."

6 The ACOG acknowledges that "cesarean deliveries that are performed for a nonreassuring fetal heart rate pattern do not necessarily preclude the use of regional anesthesia." 14

In patients with chronic fetal stress, either regional or general anaesthesia can be administered safely. In contrast, general anaesthesia is often preferred in cases of dire distress (e.g., placental abruption, prolonged fetal bradycardia, catastrophic uterine rupture). But many cases with nonreassuring fetal heart rate patterns are intermediate between chronic fetal stress and dire distress. Indeed, the term "fetal distress" is imprecise and nonspecific. Regional anaesthesia is appropriate in many of these cases. Marx et al. ${ }^{15}$ reported good results with the use of spinal and epidural anaesthesia in parturients with modest fetal distress (i.e., mean scalp capillary blood $\mathrm{pH}=7.20$ ). The following reflects my present practice:

1 I encourage the early use of epidural anaesthesia in patients at increased risk for operative delivery. Such cases include, but are not limited to, patients with breech presentation, multiple gestation, preeclampsia, diabetes, selected forms of cardiac disease, and/or a history of intrauterine fetal stress (e.g., intrauterine growth retardation, oligohydramnios). Early use and maintenance of epidural anaesthesia will facilitate the extension of epidural anaesthesia for emergency caesarean section. If an epidural catheter has been placed earlier, a partial level of anaesthesia already exists, and there is haemodynamic stability, extension of epidural anaesthesia is appropriate. I give a bolus of Ringer's lactate $i v$ and inject 2-chloroprocaine $3 \%$ epidurally in 5 - $\mathrm{ml}$ increments while the urethral catheter is inserted and the abdomen is prepared and draped. 2Chloroprocaine has a rapid onset and is rapidly metabolized by both mother and fetus. Further, placental transfer of 2-chloroprocaine is not increased by the presence of fetal acidosis. ${ }^{16}$ Often there will be satisfactory anaesthesia when the surgeon is ready to make the skin incision. If not, the ongoing fetal heart rate (FHR) tracing will dictate whether a delay is acceptable. One should not delay surgery in the presence of ongoing, severe fetal distress.

2 If there is partial but incomplete epidural anaesthesia, supplemental local infiltration with a dilute solution of local anaesthetic may result in satisfactory anaesthesia.

3 The use of spinal anaesthesia in the presence of severe fetal distress remains controversial. Spinal anaesthesia is associated with a more rapid onset of sympathectomy than epidural anaesthesia. Although spinal hypotension occurs less frequently in labouring women than in nonlabouring women, the incidence of maternal hypotension remains significant. However, Marx et al. ${ }^{15}$ and others have obtained good results with spinal anaesthesia in the presence of moderate fetal distress. Before using spinal anaesthesia in patients with acute fetal distress, one should ask oneself these questions: (a) Could the fetal distress be a result of a concealed abruption, with unrecognized hypovolaemia? (b) Can anaesthesia be achieved quickly, without delaying surgery? (c) Can hypotension be avoided? (d) If severe hypotension should occur, does the surgeon have sufficient skill to accomplish rapid delivery?

4 One should continue to monitor the FHR after transfer of the parturient to the delivery room. When possible one should continue FHR monitoring until delivery, preferably via a scalp ECG electrode. Fetal heart rate monitoring may facilitate the use of regional anaesthesia. For example, if the FHR abnormality resolves, one may wait for extension of epidural anaesthesia, or one may administer spinal or epidural anaesthesia de novo. Further, FHR monitoring may guide management in cases of failed intubation. If the anaesthetist is unable to intubate the trachea and if there is no evidence of ongoing, severe fetal distress, both the anaesthetist and the obstetrician will have greater confidence in a decision to awaken the patient and proceed with an alternate anaesthetic technique. On the other hand, if one is unable to intubate the patient, and if there is evidence of ongoing, severe fetal distress, one may decide to provide general anaesthesia via face mask ventilation, and proceed with Caesarean section.

5 In the absence of satisfactory regional anaesthesia and in the presence of ongoing, severe fetal distress, rapid sequence induction of general anaesthesia is usually indicated for Caesarean section. However, one should not indiscriminately perform rapid-sequence induction of general anaesthesia in these patients. A history and/ or suspicion of difficult intubation should prompt performance of either awake intubation or regional anaesthesia, despite the presence of fetal distress. One should not endanger the mother in order to deliver a distressed fetus.

6 Rarely, if there is a contraindication to rapid sequence induction of general anaesthesia, one may use local infiltration as the primary anaesthetic technique. In 
those cases, I recommend that the obstetrician use either 2-chloroprocaine $1.0 \%$ or lidocaine $0.5 \%$.

7 Technique of general anaesthesia for fetal distress:

(a) I do not administer a defasciculating dose of muscle relaxant, although it is acceptable practice to do so.

(b) Accomplish denitrogenation. Administration of $100 \%$ oxygen for three to five minutes is ideal, but in situations of severe fetal distress, it is acceptable to ask the mother to take four vital capacity breaths of $100 \%$ oxygen before induction of general anaesthesia. ${ }^{17}$

(c) Apply cricoid pressure.

(d) For induction, either thiopentone $\left(4 \mathrm{mg} \cdot \mathrm{kg}^{-1}\right)$ or ketamine $\left(1 \mathrm{mg} \cdot \mathrm{kg}^{-1}\right)$ is satisfactory. No laboratory or clinical data clearly favour one agent over the other.

(e) Succinylcholine $\left(1.0-1.5 \mathrm{mg} \cdot \mathrm{kg}^{-1}\right)$ remains the muscle relaxant of choice in most parturients.

(f) Insert and confirm the proper placement of a cuffed endotracheal tube.

(g) Continue to administer a high concentration of inspired oxygen (i.e., 50 to $70 \%$ ) unit delivery.

(h) Administer a low concentration of a potent halogenated agent (i.e., approximately $0.6 \mathrm{MAC}$ of halothane, enflurane, or isoflurame) to reduce the incidence of maternal awareness. Mokriski and Malinow ${ }^{18}$ recently noted no difference in the frequency or severity of umbilical arterial or venous blood acidosis after the administration of isoflurane or halothane during general anaesthesia for emergency Caesarean delivery of a distressed fetus.

(i) After delivery the anaesthetic considerations do not differ from those for other Caesarean sections. Typically I increase the inspired concentration of nitrous oxide to $70 \%$ (provided that maternal oxygen saturation remains adequate) and administer a modest dose of opioid. This allows me to decrease the concentration of the potent halogenated agent, eliminating any question regarding anaesthetic contribution to uterine atony. I avoid giving agents that might result in prolonged postoperative sedation and/or amnesia (e.g., midazolam, diazepam, droperidol).

\section{Acknowledgement}

Part of the above is excerpted, with permission, from "Anesthesia for the High Risk Obstetric Patient," 1992 Annual Refresher Course Lectures, American Society of Anesthesiologists (@1992). A copy of the full text can be obtained from the American Society of Anesthesiologists, $520 \mathrm{~N}$. Northwest Highway, Park Ridge, Illinois 60068-2573.

\section{References}

1 Haverkamp DA, Thompson HE, McFee JG, Cetrulo C. The evaluation of continuous fetal heart rate monitoring in high-risk pregnancy. Am J Obstet Gynecol 1976; 125: 310-20.

2 Renou P, Chang A, Anderson I, Wood C. Controlled trial of fetal intensive care. Am J Obstet Gynecol 1976; 126: 470-6.

3 Kelso IM, Parsons RJ, Lawrence GF, Arora SS, Edmonds $D K$, Cooke ID. An assessment of continuous fetal heart rate monitoring in labor. A randomized trial. Am J Obstet Gynecol 1978; 131: 526-32.

4 Haverkamp AD, Orleans $M$, Langendoerfer $S$, McFee J, Murphy J, Thompson HE. A controlled trial of the differential effects of intrapartum fetal monitoring. Am J Obstet Gynecol 1979; 134: 399-412.

5 Wood C, Renou P, Oats J, Farrell E, Beischer N, Anderson $I$. A controlled trial of fetal heart rate monitoring in a low-risk obstetric population. Am J Obstet Gynecol 1981; 141: 527-34.

6 MacDonald D, Grant A, Sheridan-Pereira M, Boylan P, Chalmers I. The Dublin randomized controlled trial of intrapartum fetal heart rate monitoring. Am J Obstet Gynecol 1985; 152: 524-39.

7 Leveno KJ, Cunningham FG, Nelson S, et al. A prospective comparison of selective and universal electronic fetal monitoring in 34,995 pregnancies. $N$ Engl J Med 1986; 315: 615-9.

8 Luthy DA, Shy KK, Van Belle G, et al. A randomized trial of electronic fetal monitoring in preterm labor. Obstet Gynecol 1987; 69: 687-95.

9 Shy KK, Luthy DA, Bennett FC, et al. Effects of eletronic fetal heart rate monitoring, as compared with periodic auscultation, on the neurologic development of premature infants. N Engl J Med 1990; 322: 588-93.

10 Freeman RK, Roland RL (Eds.). Guidelines for Perinatal Care, 3rd ed. American Academy of Pediatrics and American College of Obstetricians and Gynecologists, 1992, p. 77.

11 American Society of Anesthesiologists. Guidelines for regional anesthesia in obstetrics. 1991.

12 American College of Obstetricians and Gynecologists. Intrapartum fetal heart rate monitoring. Technical Bulletin $\# 132,1989$.

13 Chadwick HS, Posner K, Caplan RA, Ward RJ, Cheney $F W$. A comparison of obstetric and nonobstetric anesthesia malpractice claims. Anesthesiology 1991; 74: 242-9.

14 Committee on Obstetrics: Maternal and Fetal Medicine. Anesthesia for Emergency Deliveries. American College of Obstetricians and Gynecologists, Committee Opinion \#104, 1992.

15 Marx GF, Luykx WM, Cohen S. Fetal-neonatal status 
following Caesarean section for fetal distress. Br J Anaesth 1984; 56: 1009-13.

16 Philipson EH, Kuhnert BR, Syracuse CD. Fetal acidosis, 2-chloroprocaine, and epidural anesthesia for cesarean section. Am J Obstet Gynecol 1985; 151: 322-4.

17 Norris MC, Dewan DM. Preoxygenation for cesarean section: a comparison of two techniques. Anesthesiology 1985; 62: 827-9.

18 Mokriski BK, Manilow AM. Neonatal acid-base status following general anesthesia for emergency abdominal delivery with halothane or isoflurane. J Clin Anesth 1992: 4:

97-100. 


\section{Monitorage et anesthésie en cours de détresse foetale}

David H. Chestnut MD

\section{Monitorage foetal}

Toutes les parturientes dont le foetus est en détresse devraient-elles être suivies par monitorage cardiaque foetal continu pendant le travail? Des études approfondies randomisées semblent indiquer le peu ou même l'absence totale de bénéfices pour ce type dintervention. ${ }^{\mathrm{I}-9} \mathrm{Cer}-$ taines études concluent même que le monitoring électronique continu du coeur foetal augmente le pourcentage de césariennes. ${ }^{1,3,4,7}$ La troisième édition des " Guidelines for Perinatal Care $"{ }^{10}$ énonce ce qui suit sur le monitorage du coeur foetal chez les patientes à risque: «Il a été démontré que dans le contexte dun rapport infirmière/ patiente 1:1, l'auscultation foetale intermittente répétée aux 15 minutes pendant la phase active du premier stade du travail, et aux 5 minutes pendant le deuxième stade équivalait au monitorage électronique continu de la fréquence cardiaque. "

On ne peut s'attendre toutefois à ce que les obstétriciens délaissent le monitorage électronique. Très peu de salles de travail et d'accouchement aux Etats-Unis sont capables fournir les ressources que nécessiteraient l'auscultation intermittente et minutieuse du coeur foetal. De plus, la crainte de litiges médico-légaux force de plus en plus l'obtétricien à utiliser le monitorage électronique. La plupart des études déjà mentionnés ont été réalisées dans des hôpitaux où l'épidurale était rarement administrée pendant le travail. Ces études ne pourraient donc pas s'appliquer aux parturientes en travail sous péridurale. Ni l'American Society of Anesthesiologists (ASA), ni l'American College of Obstetricians and Gynecologists (ACOG) n'ont émis de recommandations spécifiques sur le monitorage du coeur foetal "L'anesthésie régionale pour le travail et/ou l'accouchement par voie vaginale nécessitent le monitorage et l'enregistrement des signes vitaux et du coeur foetal par une personne qualifiée. Un monitorage supplémentaire approprié à la condition de la parturiente et du foetus doit être employé lorsqu'il y a une indication ". L'ACOG de son côté recommande ce qui suit: "Lorsque l'hémodynamique maternelle est perturbée, comme il arrive avec l'anesthésie de conduction ou l'hémorragie, une surveillance accrue du foetus est indiquée ". Comme on ne peut prédire quelle patiente souffrira d'hypotension pendant l'anesthésie régionale, je préfère voir mes patientes sous monitorage électronique foetal continu avant, pendant et après l'induction de l'anesthésie régionale.

\section{L'anesthésie et la détresse foetale}

L'apparition d'une détresse foetale représente un risque médical élevé autant pour la mère que pour le foetus et un risque médico-légal accru pour l'obstétricien et l'anesthésiste. Chadwick et al. ${ }^{13}$ ont revisé les réclamations pour négligence portées contre les anesthésistes pour des cas d'obstétrique et ont comparé les réclamations obstétricales aux autres réclamations. La mortalité n'a pas été différente entre les deux types de patientes et a été reconnue comme la complication majeure la plus fréquente. Toutefois, en obstétrique, les dommages cérébraux du foetus ont été presqu'aussi fréquents que la mort maternelle. Les auteurs ajoutent: "Bien que chez le nouveau-né, l'évolution finale ne mettait en cause l'anesthésie que dans la moitié des cas, les dommages cérébraux néonataux ont été la cause principale des réclamations déposées contre les anesthésistes $"{ }^{13}$

Récemment le comité de l'ACOG sur l'obstétrique a émis son opinion dans un document intitué " Anesthesia for emergency deliveries ".$^{14}$ Cette opinion qui est favorable aux anesthésites, se résume comme suit:

1 L'ACOG reconnait que l'intubation manquée et l'aspiration pulmonaire du contenu gastrique demeurent les causes les plus importantes de morbidité et de mortalité anesthésiques.

2 L'ACOG recommande que l'équipe de soins obstétricaux soit informée de la présence des facteurs qui augmentent les risques de complications associées à l'anesthésie générale et régionale en urgence.

3 Après identification des facteurs de risques, l'ACOG recommande que l'obstétricien fasse une demande de consultation ante partum à l'anesthésiste. Pour que cette recommandation soit efficace, les anesthésistes doivent établir des procédures pour en faciliter l'application.

4 L'ACOG recommande que l'obstétricien et l'anesthésiste développent des stratégies visant à minimiser le besoin d'induire l'anesthésise générale, en urgence, chez des femmes chez qui celle-ci pourrait être particulièrement dangereuse.

5 Dans les situations d'urgence obtétricales, l'ACOG reconnaît que l'état de la mère aussi bien que celui de 
l'enfant doivent être considérés. Elle ajoute: « Bien que dans certaines situations, l'anesthésie générale soit préférable à la régionale, le risque de l'anesthésie générale doit être mis en cause et comparé à ses bénéfices pour les patientes plus sujettes aux complications. "

6 L'ACOG reconnaît que les césariennes exécutées parce que le rythme cardiaque foetal est alarmant n'excluent pas nécessairement l'usage de l'anesthésie régionale.

Lorsqu'il y a stress foetal, l'anesthésie régionale comme la générale peuvent être administrées en toute sécurité. Par contre la générale est souvent préférée dans les cas d'extrême détresse (par exemple, décollement prématuré du placenta, bradycardie foetale prolongée, rupture utérine). Les anomalies du rythme foetal se situent entre la détresse foetale chronique et la détresse extrême. En réalité, le terme " détresse foetale " est ambigu et non spécifique. L'anesthésie régionale reste appropriée dans plusieurs cas. Marx et al. ${ }^{15}$ ont rapporté des bons résultats avec la rachianesthésie et l'épidurale dans les cas de détresse foetale modérée (par exemple, avec un pH capillaire à 7,20 ). Ce qui suit représente ma pratique habituelle:

1 L'induction précoce de l'anesthésie épidurale est préférée pour les parturientes susceptibles de délivrance par césarienne dans les circonstances suivantes: les présentations du siège, grossesses multiples, la préclampsie, le diabète, certaines formes de cardiopathies, des antécédents de stress foetal intrautérin (par exemple, retard de croissance, oligohydramnios). L'induction précoce de l'épidurale et son maintien permettront de la prolonger si la césarienne devient nécessaire. Avec un cathéter épidural en place, un niveau d'analgésie installé et la stabilité hémodynamique, on pourra toujours compléter pour obtenir l'anesthésie chirurgicale. On administre un bolus de lactate $i v$ et on injecte par le cathéter épidural des doses de 2-chloroprocaine $3 \%$ par palliers de $5 \mathrm{ml}$ pendant la cathétérisation de la vessie, la désinfection et la mise en place des champs. La 2-chloroprocaïne a un début d'action rapide et est métabolisée rapidement par la mère et le foetus. L'acidose foetale n'augmente pas le transfert placentaire de la 2-chloroprocaïne. ${ }^{16} \mathrm{La}$ plupart du temps l'anesthésie sera suffisante pour permettre l'incision de la peau. Sinon, le tracé du moniteur cardiaque foetal décidera si un délai est acceptable. On ne doit jamais retarder la chirurgie pendant une détresse foetale grave.

$2 \mathrm{Si}$ l'anesthésie épidurale est incomplète, l'infiltration locale d'une solution étendue d'anesthésique peut procurer une anesthésie suffisante.

$3 \mathrm{Au}$ cours de la détresse foetale grave, l'utilisation de l'anesthésie rachidienne demeure controversée. Bien que l'hypotension d'origine rachidienne survienne moins souvent pendant le travail qu'en dehors du travail, son incidence demeure importante. Cependant Marx et al. ${ }^{\text {is }}$ et d'autres rapportent de bons résultats avec ce mode d'anesthésie pendant la détresse foetale modérée. Avant de l'utiliser, il faut se demander: a) Si la détresse est causée par un décollement prématuré du placenta et une hémorragie secondaire méconnue. b) Si on peut induire l'anesthésie rapidement sans retarder la chirurgie. c) Si l'hypotension est évitable. d) Si, en face d'une hypotension grave, le chirurgien est assez habile pour extraire le bébé en catastrophe.

4 Le coeur foetal doit être monitoré après le transfert en salle d'accouchement. Quand c'est possible, on devrait en continuer le monitorage jusqu'à l'accouchement et, de préférence, par une électrode fixée au scalp. $\mathrm{Ce}$ monitorage peut guider la conduite de l'anesthésie épidurale. Par exemple, si la détresse foetale s'estompe, l'anesthésiste peut attendre que l'anesthésie épidurale soit complète, ou administrer de novo une anesthésie rachidienne ou épidurale. Le monitorage foetal peut aussi aider à la conduite de l'anesthésiste dans les cas d'intubations difficiles. S'il ne réussit pas à intuber la patiente, et que le foetus n'est pas en détresse grave, l'anesthésiste, de concert avec obstétricien, aura l'option de réveiller la patiente et de procéder à une anesthésie régionale. D'un autre côté, dans la même situation mais en face d'une détresse foetale grave, l'anesthésiste devra recourir à une anesthésie générale sous ventilation contrôlée au masque et aller de l'avant avec la césarienne.

5 En absence d'une anesthésie régionale satisfaisante, et au cours d'une détresse foetale grave, une induction à séquence rapide est indiquée pour la délivrance par césarienne. Cependant, on ne doit pas nécessairement avoir d'emblée recours à une induction à séquence rapide. Une histoire ou l'anticipation d'intubation difficile devrait inciter l'anesthésiste recourir à l'intubation vigile ou l'anesthésie régionale malgré une détresse foetale grave. On ne doit pas mettre la vie de la mère en jeu pour extraire un foetus en détresse.

6 Dans les rares cas où l'induction à séquence rapide est contre-indiquée, on peut utiliser une infiltration locale pour extraire le foetus. Alors, il est recommandé que le chirurgien infiltre de la 2-chloroprocaïne $1,0 \%$ ou de la lidocaine $0,5 \%$.

7 Technique de l'anesthésie générale pour détresse foetale:

a) Je n'administre pas de relaxant en dose préventive pour les fasiculations, bien qu'il soit acceptable de la faire.

b) Il faut dénitrogéner. L'administration d'oxygène à $100 \%$ pour 3-5 minutes est idéale, mais dans les situations où la détresse foetale est grave, il est préférable de demander à la mère d'exécuter des 
inspirations forcées d'oxygène à $100 \%$ à quatre reprises avant l'induction de l'anesthésie générale.

c) Appliquer une pression sur le cricoïde.

d) Le thiopental $4 \mathrm{mg} \cdot \mathrm{kg}^{-1}$ et la kétamine $1 \mathrm{mg} \cdot \mathrm{kg}^{-1}$ sont deux agents d'induction satisfaisants. Les études comparatives n'accordent de supériorité ni à l'un ni à l'autre de ces agents.

e) La succinylcholine $1,0-1,5 \mathrm{mg} \cdot \mathrm{kg}^{-1}$ demeure le myo-résolutif de choix pour intuber.

f) Continuer l'administration d'une concentration élevée d'oxygène (50-70\%).

g) Administrer une faible concentration d'agent halogéné (0,6 CAM d'halothane, enflurane ou isoflurane) pour prévenir le réveil prématuré. Mokriski et Malinow ${ }^{18}$ ont comparé l'isoflurane et l'halothane pendant la césarienne effectuée pour l'extraction du foetus en détresse; ils ne rapportent pas de différence au regard de la fréquence et de la gravité pour l'acidose ombilicale foetale veineuse ou artérielle.

h) Après la naissance, les considérations anesthésiques sont les mêmes que pour les autres césariennes. J'augmente ordinairement la concentration du protoxyde d'azote à $70 \%$ en autant que la saturation maternelle demeure adéquate. Ceci permet de dimininuer la concentration de l'agent halogéné et d'éviter ainsi l'atonie utérine. Je n'utilise pas d'agents qui produissent la sédation et l'amnésie prolongées (midazolam, diazepam et le dropéridol).

\section{Références}

(Voir page R76) 